\title{
ON 2-ADJACENCY RELATION OF TWO-BRIDGE KNOTS AND LINKS
}

\author{
ICHIRO TORISU
}

(Received 21 December 2005; revised 13 October 2007)

Communicated by C. D. Hodgson

\begin{abstract}
We give a necessary condition for a two-bridge knot or link $S(p, q)$ to be 2-adjacent to another twobridge knot or link $S(r, s)$. In particular, we show that if the trivial knot or link is 2-adjacent to $S(p, q)$, then $S(p, q)$ is trivial, that if $S(p, q)$ is 2-adjacent to its mirror image, then $S(p, q)$ is amphicheiral, and that for a prime integer $p$, if $S(p, q)$ is 2-adjacent to $S(r, s)$, then $S(p, q)=S(r, s)$ or $S(r, s)=S(1,0)$.
\end{abstract}

2000 Mathematics subject classification: 57M25.

Keywords and phrases: $n$-adjacent, two-bridge knot, link, Dehn surgery.

\section{Introduction}

Let $K$ and $K^{\prime}$ be knots in $S^{3}$. We say that $K$ is $n$-adjacent to $K^{\prime}$ for some $n \in \mathbf{N}$ if $K$ admits a diagram containing $n$ (generalized) crossings such that changing any nonempty subset of them yields a diagram of $K^{\prime}$. We write $K \stackrel{n}{\longrightarrow} K^{\prime}$. By definition, $K \stackrel{n}{\longrightarrow} K^{\prime}$ implies that $K \stackrel{n^{\prime}}{\longrightarrow} K^{\prime}$ for all $0<n^{\prime} \leq n$. We remark that if $K \stackrel{n}{\longrightarrow} K^{\prime}$, all of the finite type invariants of $K$ and $K^{\prime}$ of orders at most $n-1$ agree. For example, both the trefoil knot and the figure-eight knot are 2-adjacent to the trivial knot $O$ (Figure 1). In fact, if $K$ is a two-bridge knot or genus-one knot, $K \stackrel{2}{\longrightarrow} O$ implies that $K$ is a two-bridge knot of the form $S(p, q)$ and $p / q=\left[2 q_{1}, 2 q_{2}\right]$ in Conway's notation (see [9, Theorem 1.1; 4, Theorem 5.1]). In [5], Kalfagianni and Lin proved that if $K \stackrel{n}{\longrightarrow} K^{\prime}$ and $g(K)>g\left(K^{\prime}\right)$, then $n \leq 6 g(K)-3$, where $g(\cdot)$ is the Seifert genus. Note that in the case where $K^{\prime}$ is trivial, this theorem was first obtained by Howards and Luecke in [2]. On the other hand, in [3] Kalfagianni recently proved that if $K^{\prime}$ is a fibred knot, $K \stackrel{2}{\longrightarrow} K^{\prime}$ implies $g(K)>g\left(K^{\prime}\right)$ or $K=K^{\prime}$. It follows

(c) 2008 Australian Mathematical Society 1446-7887/08 \$A2.00 + 0.00 

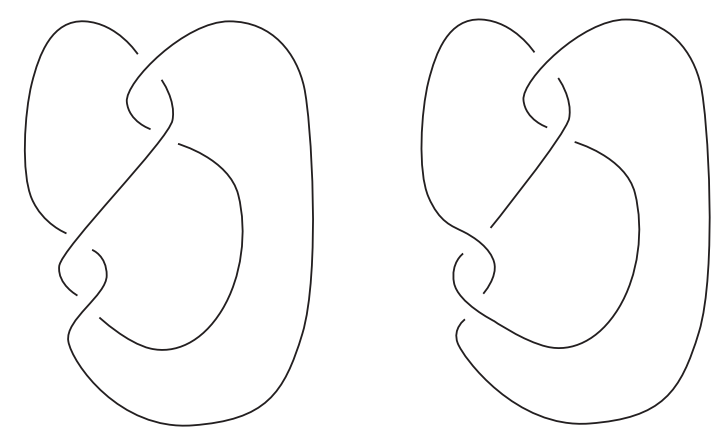

FIGURE 1. Two-bridge knots 2-adjacent to the trivial knot.

that the trivial knot is 2-adjacent to neither the trefoil knot nor the figure-eight knot [3, Remark 7.5]. Thus, $n$-adjacency is not an equivalence relation on the set of knots.

We can naturally extend the definition of $n$-adjacency to a link $L$ in $S^{3}$ with more than one component (see [10,11, 12]). In [12], Tsutsumi proved that if $L$ is 2-adjacent to a trivial link, then $L$ is a boundary link. The author does not know whether the converse is true. That is, it is a problem whether there is a nonboundary link $L$ such that a trivial link is 2 -adjacent to $L$.

In this paper, we give a necessary condition for a two-bridge knot or link $S(p, q)$ to be 2-adjacent to another two-bridge knot or link $S(r, s)$ (Theorem 1). In particular, we show that if the trivial knot or link is 2-adjacent to $S(p, q)$, then $S(p, q)$ is trivial, that if $S(p, q)$ is 2-adjacent to its mirror image, then $S(p, q)$ is amphicheiral, and that for a prime integer $p$, if $S(p, q)$ is 2-adjacent to $S(r, s)$, then $S(p, q)=S(r, s)$ or $S(r, s)=S(1,0)$. To prove our results, we use double-cover and Dehn surgery techniques as discussed in $[7,8,9,11]$. Therefore the main ingredients of the proof are the Montesinos trick [6] and the cyclic surgery theorem of Culler et al. [1].

\section{The statement of result}

Let $L$ be a link in $S^{3}$. If the number of components of $L$ is one (that is, $L$ is a knot), we may denote $L$ by $K$. A generalized crossing of order $t \in \mathbf{Z}$ on a diagram of $L$ is a set $C$ of $|t|$ twist crossings on two strings that inherit opposite orientations from any orientation of $L$. If $L^{\prime}$ is obtained from $L$ by changing all of the crossings in $C$ simultaneously, we say that $L^{\prime}$ is obtained from $L$ by a generalized crossing change of order $t$ (see Figure 2). Note that if $|t|=1, L$ and $L^{\prime}$ differ by an ordinary crossing change while if $t=0$, then $L=L^{\prime}$. Throughout this paper, we assume that $t \neq 0$.

Let $S(p, q)$ be a two-bridge knot or link whose two-fold branched cover is the lens space $L(p, q)$, where $p, q$ are relatively prime integers. Then $S(p, q)$ is a twocomponent link for an even $p$ and a knot for an odd $p$. In particular $S(0,1)$ is the two-component trivial link and $S(1,0)$ is the trivial knot. 




FIGURE 2. A generalized crossing change of order -3 .

Recall that $L$ is $n$-adjacent to $L^{\prime}$ (denoted by $L \stackrel{n}{\longrightarrow} L^{\prime}$ ), if $L$ admits a diagram containing $n$ generalized crossings such that changing any nonempty subset of them yields a diagram of $L^{\prime}$.

TheOREM 1. Suppose that $S(p, q)$ is 2-adjacent to $S(r, s)$. Then:

(i) $\quad p$ is factorized by $r$; and

(ii) if $p=r, S(p, q)=S(r, s)$.

COROLLARY 2. We have the following.

(i) Suppose that the trivial knot is 2-adjacent to $S(p, q)$. Then $S(p, q)$ is the trivial knot $S(1,0)$.

(ii) Suppose that the two-component trivial link is 2-adjacent to $S(p, q)$. Then $S(p, q)$ is the trivial link $S(0,1)$.

(iii) Suppose that $S(p, q)$ is 2-adjacent to $S(p,-q)$. Then $S(p, q)=S(p,-q)$, that is, $S(p, q)$ is amphicheiral.

(iv) For a prime integer $p$, suppose that $S(p, q)$ is 2-adjacent to $S(r, s)$. Then $S(p, q)=S(r, s)$ or $S(r, s)=S(1,0)$.

\section{Proof of Theorem 1}

Let $N(K)$ be a regular neighbourhood of a knot $K$ in a closed orientable 3-manifold $M$, with $\mu$ a meridian for $N(K)$ and let $E(K)=M-\operatorname{int} N(K)$ be the exterior of $K$ in $M$. Now let $K(\gamma)$ denote the manifold obtained by attaching a solid torus $V$ to $E(K)$ so that a curve of slope $\gamma$ on $\partial E(K)$ bounds a disc in $V$, where $\gamma$ indicates the isotopy class of an essential simple closed curve on the 2-torus. We say that $K(\gamma)$ is the result of $\gamma$-Dehn surgery on $K$. Dehn surgery on a 2-component link $K \cup K^{\prime}$ is also defined in the same way and denoted by $K(\gamma) \cup K^{\prime}\left(\gamma^{\prime}\right)$ for slopes $\gamma$ and $\gamma^{\prime}$ of $K$ and $K^{\prime}$, respectively. For two slopes $\gamma$ and $\delta$ in $\partial E(K)$, let $\Delta(\gamma, \delta)$ be their minimal geometric intersection number.

First of all, the Montesinos trick in [6] connects a generalized crossing change with Dehn surgery. 
LEMMA 3 [6]. Let $L$ and $L^{\prime}$ be links in $S^{3}$ and let $M_{L}$ and $M_{L^{\prime}}$ be the two-fold branched covering spaces of $S^{3}$ along $L$ and $L^{\prime}$, respectively. Suppose that the result of a generalized crossing change of order $t$ on $L$ is $L^{\prime}$. Then $M_{L^{\prime}}$ is obtained by $\gamma$-Dehn surgery on some knot $K$ in $M_{L}$, where $\Delta(\gamma, \mu)=2|t|$. Moreover, $N(K)$ is obtained by the lift of a crossing ball as illustrated in Figure 2 by the dotted circle.

A torus knot in a lens space is a knot isotopic to a Heegaard torus of the lens space. For $i=1,2$, let $V_{i}$ be a solid torus standardly embedded in $S^{3}$ and let $\mu_{i}$ and $\lambda_{i}$ be a meridian and a longitude of $V_{i}$, respectively. Let $h$ be an orientationreversing homeomorphism from $\partial V_{1}$ to $\partial V_{2}$ such that $h\left(\mu_{1}\right)=s \mu_{2}+r \lambda_{2}$. Then the space $V_{1} \bigcup_{h} V_{2}$ obtained from $V_{1}$ and $V_{2}$ by identifying their boundaries by $h$ is the lens space $L(r, s)$. Let $C_{m, n}$ be a $(m, n)$-curve on $\partial V_{1}$, that is, a simple closed curve which is isotopic to $m \mu_{1}+n \lambda_{1}$. Let $a, b$ be integers such that $r b-s a=1$. Then we may assume $h\left(\lambda_{1}\right)=b \mu_{2}+a \lambda_{2}$ and $C_{m, n}$ is equal to $(s m+b n) \mu_{2}+(r m+a n) \lambda_{2}$ on $\partial V_{2}=\partial V_{1}$. Note that if $n=0$ or $r m+a n=0$, then $C_{m, n}$ is the trivial knot in $L(r, s)$. Then it is not hard to see that every torus knot in $L(r, s)$ is isotopic to some $C_{m, n}$. We may push $C_{m, n}$ into int $V_{1}$. Then, for a slope $\gamma$ on $\partial N\left(C_{m, n}\right)$, using the usual preferred meridian-longitude coordinates of $\partial N\left(C_{m, n}\right)$ in $V_{1} \subset S^{3}$, we identify $\gamma$ with $c / d \in \mathbf{Q} \cup\{\infty\}$ where $c$ and $d$ are relatively prime.

We need the following calculations from [7] and [8].

THEOREM 4 [7]. The space $C_{m, n}(c / d)$ is homeomorphic to a lens space if and only if there is a pair of coprime integers $m^{\prime}, n^{\prime}$ such that:

(i) $C_{m, n}$ is isotopic to $C_{m^{\prime}, n^{\prime}}$ in $L(r, s)$;

(ii) $c=d m^{\prime} n^{\prime} \pm 1$; and

(iii) $C_{m^{\prime}, n^{\prime}}(c / d)$ is orientation-preserving homeomorphic to $L\left(d a n^{\prime 2}+r\left(d m^{\prime} n^{\prime} \pm\right.\right.$ $\left.1), d b n^{\prime 2}+s\left(d m^{\prime} n^{\prime} \pm 1\right)\right)$, where $a, b$ are as above for $C_{m^{\prime}, n^{\prime}}$.

Corollary 5 [8, Proof of Theorem 2.2]. Let $K_{T}$ be a torus knot in $L(r, s)$. Suppose that $K_{T}(\gamma)$ is orientation-preserving homeomorphic to $L(r, s)$ for some slope $\gamma$ with $\Delta(\gamma, \mu) \geq 2$. Then $K_{T}$ is the trivial knot, that is, $K_{T}$ bounds a disc in $L(r, s)$.

Proof of Corollary 5. Put $K_{T}=C_{m, n}$ and $\gamma=c / d$ with $|d| \geq 2$. By Theorem 4 and the classification of lens spaces, we conclude that for coprime $m^{\prime}, n^{\prime}, r=$ $d a n^{\prime 2}+r\left(d m^{\prime} n^{\prime} \pm 1\right)$ and either $s$ is congruent to $d b n^{\prime 2}+s\left(d m^{\prime} n^{\prime} \pm 1\right)$ modulo $r$, or $s\left(d b n^{\prime 2}+s\left(d m^{\prime} n^{\prime} \pm 1\right)\right)$ is congruent to 1 modulo $r$. Then an elementary congruence argument shows that $n^{\prime}=0$ or $r m^{\prime}+a n^{\prime}=0$. Therefore, $C_{m^{\prime}, n^{\prime}}$ and, hence, $C_{m, n}$ is trivial.

Proposition 6. Suppose that $S(p, q)$ is 2-adjacent to $S(r, s)$ by two generalized crossing changes of orders $t_{1}$ and $t_{2}$. Then there is a two-component link $K_{1} \cup K_{2}$ in $L(r, s)$ such that $K_{1}\left(\gamma_{1}\right)$ and $K_{2}\left(\gamma_{2}\right)$ are orientation-preserving homeomorphic to $L(r, s)$ and $K_{1}\left(\gamma_{1}\right) \cup K_{2}\left(\gamma_{2}\right)$ is orientation-preserving homeomorphic to $L(p, q)$, where each slope $\gamma_{i}$ satisfies $\Delta\left(\gamma_{i}, \mu\right)=2\left|t_{i}\right|$. Moreover, each $K_{i}$ is the trivial knot in the original $L(r, s)$ and a torus knot in $K_{j}\left(\gamma_{j}\right)=L(r, s)(i \neq j)$. 
Proof of Proposition 6. By definition, there are two generalized crossings of orders $t_{1}, t_{2}$ in a diagram of $S(p, q)$ such that both each generalized crossing change and simultaneous generalized crossing changes yield $S(r, s)$. The two-fold cover of $S(r, s)$ in $S^{3}$ is $L(r, s)$. Therefore, by repeated use of Lemma 3, there exists a 2-component link $K_{1} \cup K_{2}$ in $L(r, s)$ such that $K_{1}\left(\gamma_{1}\right)=K_{2}\left(\gamma_{2}\right)=L(r, s)$ and $K_{1}\left(\gamma_{1}\right) \cup K_{2}\left(\gamma_{2}\right)=L(p, q)$, where $\Delta\left(\gamma_{i}, \mu\right)=2\left|t_{i}\right|(i=1,2)$. Moreover, since $\Delta\left(\gamma_{i}, \mu\right)=2\left|t_{i}\right| \geq 2$, by the cyclic surgery theorem of Culler et al. [1], the exterior spaces $E\left(K_{1}\right)$ and $E\left(K_{2}\right)$ are Seifert fibred manifolds. However, as in [7, Lemma 4] this implies that $K_{1}$ and $K_{2}$ are fibres for some Seifert fibrations of $L(r, s)$. Moreover, it is well-known that any fibre for a Seifert fibration of a lens space is isotopic to some torus knot (see, for example, [7]). By Corollary 5, it follows that $K_{1}$ and $K_{2}$ are trivial in the original $L(r, s)$, completing the proof of Proposition 6.

We are ready to prove Theorem 1 .

Proof of Theorem 1 . Let $K_{T}$ be a torus knot $K_{1}$ in $K_{2}\left(\gamma_{2}\right)=L(r, s)$ as in the last statement of Proposition 6. Since $K_{2}$ is trivial, $K_{T}$ in $K_{2}\left(\gamma_{2}\right)=L(r, s)$ may be considered as a full-twisted $K_{1}$ along some disc spanned by $K_{2}$ in the original $L(r, s)$. Note that a full-twist operation for a circle does not change the homotopy class in $L(r, s)$. Hence, $K_{T}$ is homotopically trivial in $K_{2}\left(\gamma_{2}\right)=$ $L(r, s)$. Suppose that $K_{T}$ is isotopic to $C_{m, n}$. Then it follows that $n=0$ or $n=r$. Applying Theorem 4 to $K_{1}\left(\gamma_{1}\right) \cup K_{2}\left(\gamma_{2}\right)=L(p, q)$ and $d=2 t_{1}$, we have $L(p, q)=L\left(2 t_{1} a n^{\prime 2}+r\left(2 t_{1} m^{\prime} n^{\prime} \pm 1\right), 2 t_{1} b n^{\prime 2}+s\left(2 t_{1} m^{\prime} n^{\prime} \pm 1\right)\right)$ for coprime $m^{\prime}, n^{\prime}$. Since $C_{m, n}$ is isotopic to $C_{m^{\prime}, n^{\prime}}$, we have $n^{\prime}=0$ or $n^{\prime}=r \neq 0$. If $n^{\prime}=0$, then the statement of Theorem 1 apparently holds. So put $n^{\prime}=r \neq 0$. Then since $p=2 t_{1} a r^{2}+$ $r\left(2 t_{1} m^{\prime} r \pm 1\right)=r\left(2 t_{1} a r+2 t_{1} m^{\prime} r \pm 1\right)$ and $2 t_{1} a r+2 t_{1} m^{\prime} r \pm 1 \neq 0, p$ is factorized by $r$, verifying (i). Further suppose that $p=r$, then $r=r\left(2 t_{1} a r+2 t_{1} m^{\prime} r \pm 1\right)$. Therefore, $2 t_{1} a r+2 t_{1} m^{\prime} r \pm 1=1$ and, thus, $2 t_{1} r\left(a+m^{\prime}\right)=0$ or 2 . Since $t_{1} \neq 0$, it follows that $r\left(a+m^{\prime}\right)$ is 0 or 1 . If $r\left(a+m^{\prime}\right)=r a+m^{\prime} n^{\prime}=0$, then $L(p, q)=L(r, s)$ because in this case $C_{m^{\prime}, n^{\prime}}$ is the trivial knot in $L(r, s)$. If $r\left(a+m^{\prime}\right)=1$, then $r=1$ and $L(p, q)=L(r, s)=L(1,0)$, verifying (ii). This completes the proof of Theorem 1.

REMARK 7. We make the following remarks.

(i) It is still a problem to decide on the exact pairs of $(p, q)$ and $(r, s)$ such that $S(p, q) \stackrel{2}{\longrightarrow} S(r, s)$.

(ii) At the time of writing, the author was not aware of any example of a nontrivial knot or link $L$ such that a trivial knot or link is 2-adjacent to $L$.

\section{References}

[1] M. Culler, C. Gordon, J. Luecke and P. Shalen, 'Dehn surgery on knots', Ann. of Math. (2) 125 (1987), 237-300.

[2] H. Howards and J. Luecke, 'Strongly n-trivial knots', Bull. London Math. Soc. 34 (2002), $431-437$. 
[3] E. Kalfagianni, 'Crossing changes of fibered knots', Preprint.

[4] E. Kalfagianni and X.-S. Lin, 'Knot adjacency and satellites', Topology Appl. 138 (2004), 207-217.

[5] - 'Knot adjacency, genus and essential tori', Pacific J. Math. 228 (2006), 251-275.

[6] J. M. Montesinos, 'Surgery on links and double branched coverings of $S^{3}$, Ann. of Math. Stud. 84 (1975), 227-259.

[7] I. Torisu, 'The determination of the pairs of two-bridge knots or links with Gordian distance one', Proc. Amer. Math. Soc. 126 (1998), 1565-1571.

[8] , 'On nugatory crossings for knots', Topology Appl. 92 (1999), 119-129.

[9] - 'On strongly $n$-trivial 2-bridge knots', Math. Proc. Cambridge Philos. Soc. 137 (2004), 613-616.

[10] ' 'A note on strongly $n$-trivial links', J. Knot Theory Ramifications 14 (2005), 565-569.

[11] , 'Two-bridge links with strong triviality', Tokyo J. Math. 29 (2006), 233-237.

[12] Y. Tsutsumi, 'Strongly $n$-trivial links are boundary links', Tokyo J. Math. 30 (2007), 343-350.

ICHIRO TORISU, Naruto University of Education, 748, Nakajima, Takashima, Naruto-cho, Naruto-shi, 772-8502, Japan

e-mail: torisu@naruto-u.ac.jp 